\title{
Corrigendum to "Clinical Outcomes of Critically Ill Patients Using Inhaled Nitric Oxide (iNO) during Intrahospital Transport"
}

\author{
Leonid Koyfman, ${ }^{1}$ Omri Simchon, ${ }^{1}$ Anna Koyfman, ${ }^{2}$ Shoshana Moshinsky, ${ }^{3}$ \\ Benjamin Fredrick Gruenbaum, ${ }^{4}$ Ron Gal, ${ }^{1}$ Michael Friger ${ }^{(D)},{ }^{5}$ Natan Arotsker, \\ Alexander Zlotnik, ${ }^{1}$ Moti Klein, ${ }^{1}$ and Evgeni Brotfain $\mathbb{D}^{1}$ \\ ${ }^{1}$ Department of Anesthesiology and Critical Care, General Intensive Care Unit, Soroka Medical Center, \\ Ben-Gurion University of the Negev, Beer Sheva, Israel \\ ${ }^{2}$ Department of Radiology, Meir Medical Center, Kfar Saba, Israel \\ ${ }^{3}$ Department of Internal Medicine, Soroka Medical Center, Ben-Gurion University of the Negev, Beer Sheva, Israel \\ ${ }^{4}$ Department of Anesthesiology and Perioperative Medicine, Mayo Clinic, Jacksonville, FL, USA \\ ${ }^{5}$ Department of Public Health, Faculty of Health Sciences, Ben-Gurion University of the Negev, Beer Sheva, Israel
}

Correspondence should be addressed to Evgeni Brotfain; bem1975@gmail.com

Received 1 June 2021; Accepted 1 June 2021; Published 21 June 2021

Copyright ( $\odot 2021$ Leonid Koyfman et al. This is an open access article distributed under the Creative Commons Attribution License, which permits unrestricted use, distribution, and reproduction in any medium, provided the original work is properly cited.

In the article titled "Clinical Outcomes of Critically Ill Patients Using Inhaled Nitric Oxide (iNO) during Intrahospital Transport" [1], there was a spell error in author Shoshana Moshinsky's name in the author list, where "Shoshana Mushaev" should have read "Shoshana Moshinsky." This is corrected as shown in the author details above.

\section{References}

[1] K. Leonid, K. Anna, S. Mushaev et al., "Clinical outcomes of critically Ill patients using inhaled nitric oxide (iNO) during intrahospital transport," Research and Practice, vol. 2021, Article ID 6633210, 6 pages, 2021. 